\title{
Poster Abstract: Enabling Identity-Aware Tracking by Vision-RFID Fusion
}

\author{
Haofan Cai \\ University of California Santa Cruz \\ Santa Cruz, CA, USA \\ hcai10@ucsc.edu
}

\author{
Chen Qian \\ University of California Santa Cruz \\ Santa Cruz, CA,USA \\ cqian12@ucsc.edu
}

\begin{abstract}
Person identification and tracking (PIT) is an essential research topic in computer vision (CV). A CV-based system typically needs to identify, locate, and track persons appearing in its sight. In this work, we propose RFTrack, an RFID and CV fushion system that enables cameras in public areas (like surveillance cameras) to 'recognize' the physicalidentity(ID) of persons in the fields of view and track the persons with specific IDs with no training efforts. By asking the users to perform a simple authentication, the system will be aware of the targets' IDs in its sensing range. Later through comparing the motion trajectories derived from both camera videos and RF signal, we can associate RFIDtagged human objects in videos with their physical IDs. A preliminary study conducted shows that RFTrack can actively identify and track the RFID-tagged target objects using commercial RFID devices and cameras, in complex indoor environments where various multipath reflectors exist.
\end{abstract}

\section{CCS CONCEPTS}

- Human-centered computing $\rightarrow$ Human computer interaction (HCI), Ubiquitous and mobile computing.

\section{KEYWORDS}

Wireless sensor network, RFID, human tracking

\section{ACM Reference Format:}

Haofan Cai and Chen Qian. 2020. Poster Abstract: Enabling Identity-Aware Tracking by Vision-RFID Fusion. In The 18th ACM Conference on Embedded Networked Sensor Systems (SenSys '20), November 16-19, 2020, Virtual Event, Japan. ACM, New York, NY, USA, 2 pages. https://doi.org/10.1145/3384419.3430418

\section{INTRODUCTION}

With the advent of Internet of Things (IoT), the inexpensive, network-enabled cameras have being widely deployed in public places like airports and enabled a wide range of real-world applications such as visual surveillance, suspicious activity and anomaly detection and crowd behavior analysis. These cameras record every event in their view, making

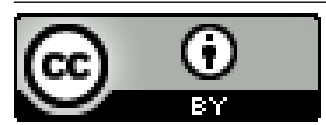

This work is licensed under a Creative Commons Attribution International 4.0 License. SenSys '20, November 16-19, 2020, Virtual Event, Japan (C) 2020 Copyright held by the owner/author(s).

ACM ISBN 978-1-4503-7590-0/20/11.

https://doi.org/10.1145/3384419.3430418

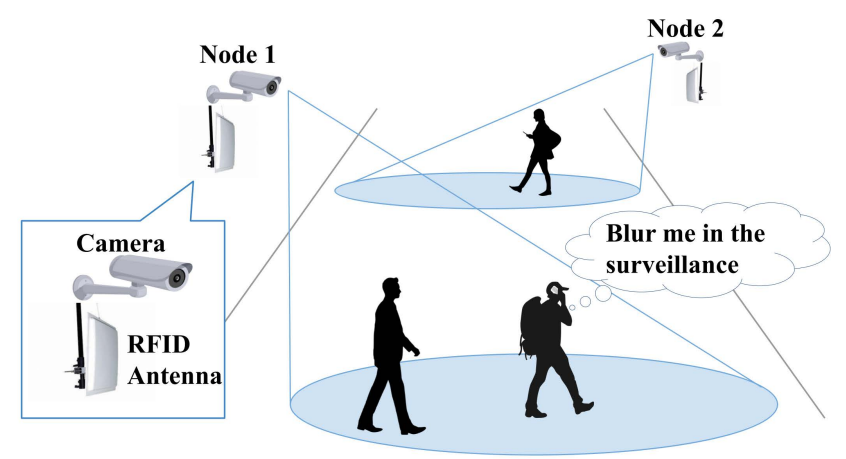

Figure 1: Application scenario

their videos valuable evidence[3]. However, sharing of video evidences, even for the common good, creates some threats to the privacy of individuals in the footage. Though people are often willing to release their video evidences to the public, especially when having captured unusual scenes such as traffic accidents, crime scenes, etc, sometimes they may also want to be blurred in the surveillance cameras for privacy purposes. For example, as shown in Fig. 1, suppose that in an office building where surveillance cameras are pervasively deployed and people come and go in all directions. If one of these pedestrians wants to remove her trajectory in the video, then how to accurately identify this pedestrian's ID and track her movement become challenging problems.

Motivated by the aforementioned scenario, we propose an interesting research question:Is it possible for cameras to 'recognize' the physical-identity of person in the fields of view and track the person with specific ID with least or even no training efforts? In other words, we aims to (i) identify multiple mobile persons in a camera with their IDs as well as (ii) track the movements of these persons as long as they are in camera's view . For simplicity, we use the term 'target' to refer to the person who would like to get herself blurred in camera. This raises a challenging task for vision system as the camera needs to first find out which object(person) in its field of view makes this request, only then it is able to track this target and keep it blurred in the following video stream until the target leaves its view. And because of the unavailability of any direct communication between people and camera, it is quite hard for vision system to automatically associate the real identity of a person with the objects in video.

In this study, we argue that the wireless communication between the target and the reader through the $\mathrm{RF}$ channel can essentially assist the visual channel to actively find the mobile 


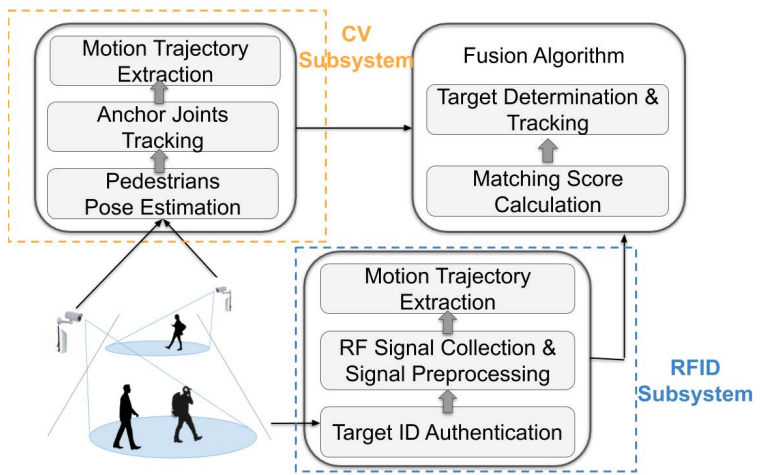

Figure 2: System Overview

target without any training. We propose RFTrack, a finegrained identifying and tracking schema for tagged targets, which fuses RFID and CV together using COTS RFID devices and depth cameras. RFTrack is able to automatically detect, localize, and identify any target in the video when he/she appears in the camera and then keep tracking it, without relying on finding biological features of human.

\section{METHODOLOGY}

We show the overview of our CV-RFID fusion system in Fig. 2. RFTrack is prototyping with several nodes using commercial RFID reader, antennas and depth cameras which can transform the target's location in the recorded images to the 3D real world position. Basically, a RFTrack node consists of an omnidirectional antenna and a depth camera on top of the antenna (as shown in Fig. 1) and can be deployed at different places in a building, with camera's field of view among different nodes overlapping or non-overlapping. The user who would like to be blurred in the camera's view needs to carry a RFID tag with her.

RFTrack mainly goes through the following steps to recognize and track the mobile targets: (i) At the user authentication phase, the user who would like to blur herself in the video will first performance inductive coupling using TagMii [1] method at authentication platform, and get her tag being recognized and authenticated by RFID subsystem. (ii) Once the user's ID gets authenticated, RFID subsystem then obtains and calibrates the phase shifts from this target tag's signal snapshots and calculates the line-of-sight(LOS) distance between the platform's antenna and tag (user). While for CV subsystem, given consecutive recorded video frames, RFTrack will estimate the moving trajectory of each object in these frames. Here we apply pose estimator AlphaPose [2] to infer poses of different persons in video frames and then leverage some specific joints to measure the moving trajectory of each target. Since we ask participants to always hold their ID tags during experiments in our study, the joints for both left and right wrists can be chosen as anchors for moving trajectory estimation. The output of pose estimator for each video frame is shown in Fig. 3. And each participant's trajectory can be easily recovered by locating the chosen anchor joints in 3D space among consecutive frame stream.

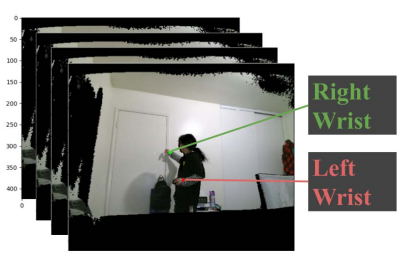

Figure 3: Output results of AlphaPose pose estimator

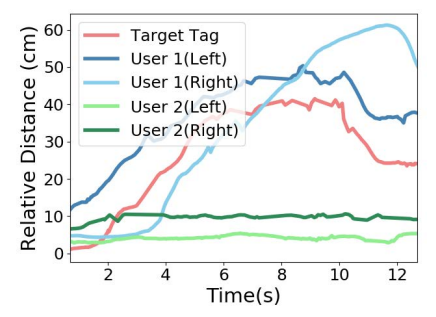

Figure 4: Relative distance trajectory recovered from CV image stream
(iii)Then for the target target, RFTrack tries to match it to one of the motion trajectories that is most likely to be the tags host. A matching score will be calculated and assigned to each motion trajectory and the tag will be matched to the specific motion trajectory with the highest matching score.

\section{EVALUATION RESULTS}

Our preliminary study compares temporal correlation between trajectory recovered from RF signal and the one obtained from camera. We recruited two female participants and conducted the experiments indoors. Both participants were asked to carry two RFID tags with her while in each experiment, only the tag hold in user 1's left hand is allowed to be authenticated at the platform. During the experiment, participants randomly wandered in the room. In Fig. 4, we show the LOS distance trajectory recovered from two users' wrist motions as well as the trajectory of the authenticated tag . The $\mathrm{X}$-axis is the time duration for the experiment, and $\mathrm{Y}$-axis is the measured LOS distance between tagged user and RFTrack node. As can be seen from the results, target tag's trajectory recovered from RFchannel (red curve) is highly correlated with the user's hand movement obtained by CV (steelblue curve).

\section{CONCLUSION}

In this paper, we present RFTrack, a vision-RFID fusion system which can enable cameras to recognize the physical IDs of persons in their views and track the targets instantly when they appear in the video frames with no training efforts. We envision that in the future design, RFTrack can be extended to resolve commonly seen multi-target-multi-camera-tracking (MTMC) problems.

\section{REFERENCES}

[1] Haofan Cai, Ge Wang, Xiaofeng Shi, Junjie Xie, Minmei Wang, and Chen Qian. 2019. When tags readeach other: Enabling lowcost and convenient tag mutual identification. In 2019 IEEE 27th International Conference on Network Protocols (ICNP). IEEE, $1-11$.

[2] Hao-Shu Fang, Shuqin Xie, Yu-Wing Tai, and Cewu Lu. 2017. RMPE: Regional Multi-person Pose Estimation. In ICCV.

[3] Hyunwoo Yu, Jaemin Lim, Kiyeon Kim, and Suk-Bok Lee. 2018. Pinto: enabling video privacy for commodity IoT cameras. In Proceedings of the 2018 ACM SIGSAC Conference on Computer and Communications Security. 1089-1101. 